\title{
Possibility for nuclear physics study based on Pelletron ac- celerator at Hanoi, Vietnam
}

\author{
Cuong Phan Viet ${ }^{1, *}$, Anh Le Tuan ${ }^{2}$, Chung Le Xuan ${ }^{3}, H a$ Nguyen Hong ${ }^{4}$, Thao Ho $\mathrm{Thi}^{4}$, and \\ Khue Pham Duc ${ }^{3}$ \\ ${ }^{1}$ Research and Development Center for Radiation Technology, Vietnam Atomic Energy Institute, Thu \\ Duc, Ho Chi Minh, Vietnam \\ ${ }^{2}$ Vietnam Atomic Energy Institute, 59 Ly Thuong Kiet, Hoan Kiem, Hanoi, Vietnam \\ ${ }^{3}$ Insitutte of Nuclear Science and Technology, Vietnam Atomic Energy Institute, Nghia Do, Hanoi \\ ${ }^{4}$ Institute of Physics, VAST, Hanoi, Vietnam
}

\begin{abstract}
In this report, overview of the new installed Pelletron 5SDH-2 accelerator at Hanoi University of Science, Vietnam and possibilities for performing astrophysics-related nuclear reactions based on this accelerator will be described. In addition to that, some preliminary simulation results for designing and optimizing the experimental set-up in studying reactions ${ }^{10} B(\alpha, p){ }^{13} C$ with projectile energies in the Gamow-window will be shown.
\end{abstract}

\section{Introduction}

Pelletron 5SHD-2 acclerator installed 2011 at Hanoi Unversity of Science (HUS) is 1.7 MV tandem electronstatic type, manufactured by National Electrostatic Corporation, USA. With dual source of negative ion by cesium sputtering (SNICS) and radio frequency ion source, the accelerator can provide beam of over wide range of ion species. The proton is acclerated to $1.7 \mathrm{MeV}$ while $\alpha$ is accelerated to $5.1 \mathrm{MeV}$. The accelerator has two experimental beam line, one for nuclear analysis and the other for ion implantation. With low energy proton beam, proton-induced X-ray emission (PIXE) analysis technique is being applied allowing to determine elemental make-up of the material or sample with sensitivity at level of ppb. Other analysis technique is Rutherford backscattering (RBS) using low energy $\alpha$, which is useful to determine the composition of a thin layer surface.

Beside three above applications, recently a program to study astrophysics-related nuclear reaction induced by low energy proton and $\alpha$ beam within Gamow window has been launched. Some reactions such ${ }^{7} B e(\alpha, \gamma){ }^{11} C$ and ${ }^{10} B(\alpha, p){ }^{13} C \ldots$ relevant to breakout from p$\mathrm{p}$ chain region $(\mathrm{A}<12)$ which affect $v p$ process [1] is concerned. In our experiment campaing, the reaction ${ }^{10} B(\alpha, p){ }^{13} C$ is firstly selected to perfom. Although knowing the cross-section of this reaction is very crucial to understand the abundant of some elements but there were only few experiments to measure this quantity for different energy of $\alpha$ so far, namely Bonner et al [2](with $\alpha$ energy: 2.16 to $5.36 \mathrm{MeV}$ ), Wilson et al [3] (with $\alpha$ energy: 2 to $8 \mathrm{MeV}$ ) and Chen et al [4] (with $\alpha$ energy: 1.4 to $5.3 \mathrm{MeV}$ ), it however, descrepancy between those experiment's data is quite big. In our planned experiment, we are going to re-measured the cross-section of

\footnotetext{
*e-mail: pvcuong@ vinatom.gov.vn
} 


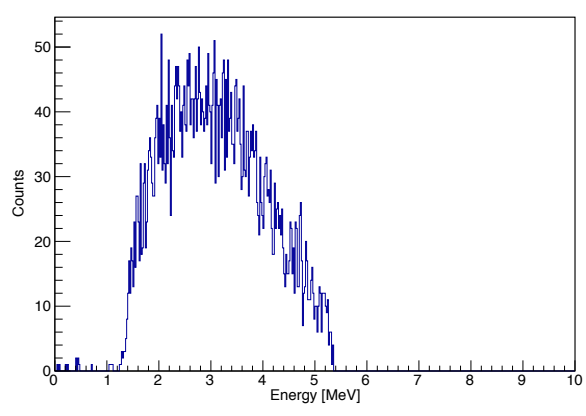

(a) G4Bertini

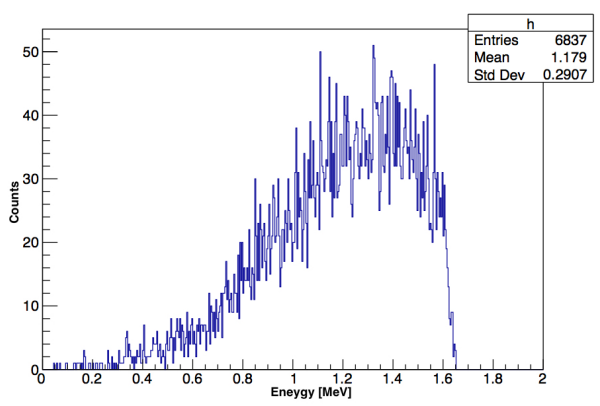

(c) G4Binary

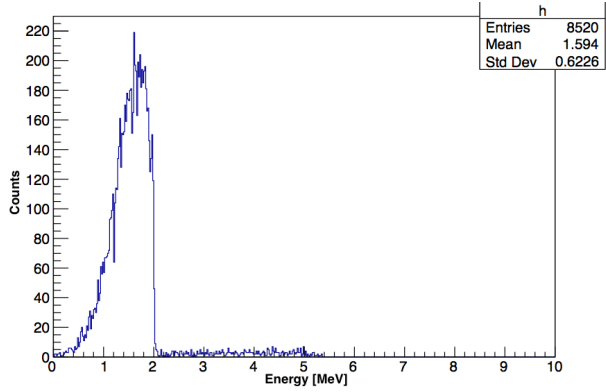

(b) G4Incl++

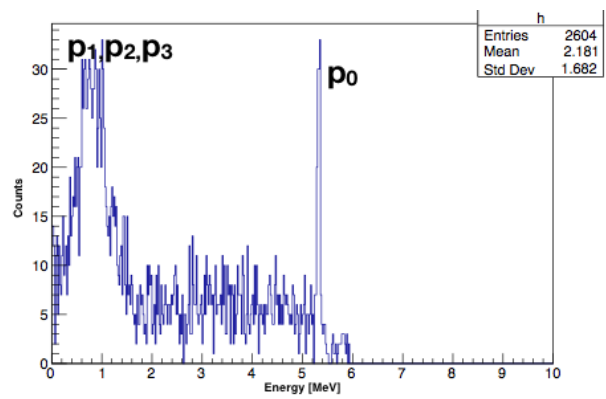

(d) G4ParticlHP

Figure 1: Proton yield from ${ }^{10} B(\alpha, p){ }^{13} C$ reaction simulated by some built-in Geant 4 models

this reaction for $\alpha$ energy lower than $3.4 \mathrm{MeV}$ down to $0.7 \mathrm{MeV}$ which covering the reaction Gamow window of $v p$-process relevance $\left(T_{9}=1-1.5\right)$. In this paper, we report the development of the Geant4-based code to simulate the reaction induced by low energy $\alpha$ which then would help to design future experiment and to analysis the data. The simulation was performed for reaction ${ }^{10} B(\alpha, p){ }^{13} C$ at $\alpha$ energy of $1.512 \mathrm{MeV}$ which corresponding to one experimental point in [4] for valiation the developed code, the charge energy spectrums by silicon detectors are aslo simulated.

\section{Geant4 simulation for ${ }^{10} B(\alpha, p){ }^{13} C$ reaction}

Geant4 has several built-in models for nuclear reaction, such as G4Bertini, G4Binary, G4Incl++, G4ParticleHP. For low energy reaction, however, these models fails to generate acceptable result for energy spectrum of proton from ${ }^{10} B(\alpha, p){ }^{13} C$ reaction (see Fig.1). Therefore, a simple model has to be implemented into Geant 4 to serve as a tool for design the experimental setup.

The implementation of a physical in Geant 4 has two modules. The first is dedicated to calculate the interaction cross-section and the second calculates the final state particle as well as their kinematic distribution. The talysxcross class was implemented based on G4VCrossSectionDataSet class. Its role is to take the cross-sections culcualted by Talys1.9 [5] and put them into Geant 4 code. Figure 2 shows the total reaction cross-section for $\alpha+{ }^{10,11} B$ obtained by Talys. 


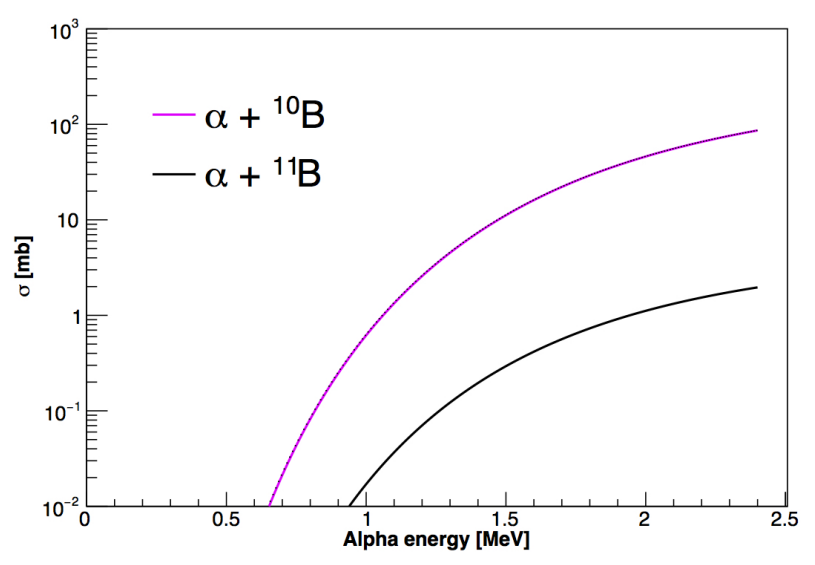

Figure 2: Total cross-section of $\alpha+{ }^{10,11} B$ reactions calculated by Talys-1.9 [5]

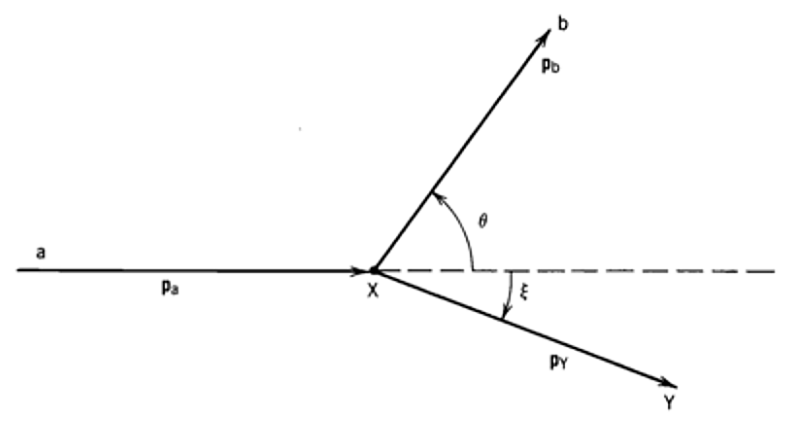

Figure 3: Basic reaction geometry for $a+X \rightarrow b+Y$

The seconde module, which inherits from G4HadronicInteraction base clase, generates the kinematics distribution for final state particles. Figure 3 expresses a simple geometry for $a+X \rightarrow b+Y$. By using conservation of total energy law and the conservation of momentum law, One obtains the relationship between kinetic energy and angle of outgoing particle:

$$
T_{b}^{1 / 2}=\frac{\left(m_{a} m_{b} T_{a}\right)^{1 / 2} \cos \theta \pm\left\{m_{a} m_{b} T_{a} \cos ^{2} \theta+\left(m_{Y}+m_{b}\right)\left[m_{Y} Q+\left(m_{Y}-m_{a}\right) T_{a}\right]\right\}^{1 / 2}}{m_{Y}+m_{b}}
$$

where m's are the rest masses, and T's are kinetic energies.

A Geant4 built-in class named G4TwoBodyAngularDist was used for generating the angular distribution of final particles. The relative yields for $\mathrm{p}_{0}, \mathrm{p}_{1}, \mathrm{p}_{2}, \mathrm{p}_{3}$ from $10 \mathrm{~B}(\mathrm{a}, \mathrm{p})^{13} \mathrm{C}$ reaction were estimated from experimental results obtained by Chen et al [4] (see in Fig.4).

\section{Simulation results and discussion}

In our simulation, the target is natural Boron $\left(19 \%{ }^{10} \mathrm{~B}, 80.1 \%{ }^{11} \mathrm{~B}\right)$ with density of $2.37 \mathrm{~g} / \mathrm{cm}^{3}$ and dimension of $0.5 \mathrm{~cm} \times 0.5 \mathrm{~cm}$. The target is titled $45^{\circ}$ relative to the beam axis. Four silicon 


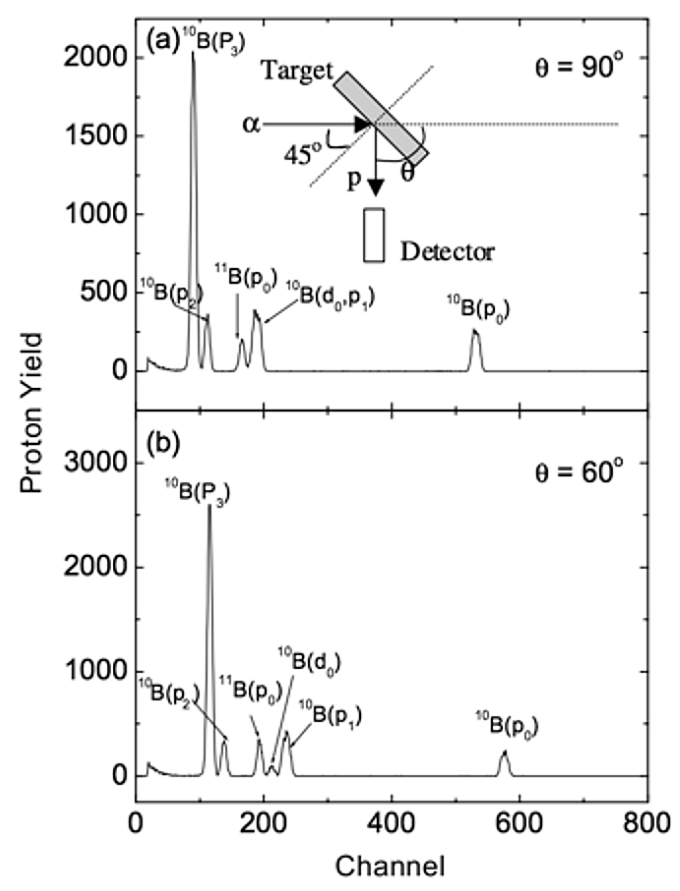

Figure 4: Energy spectrum of proton from the $10 \mathrm{~B}(\mathrm{a}, \mathrm{p}){ }^{13} \mathrm{C}$ reaction using a natural abundance boron thin film $\left({ }^{10} \mathrm{~B} 19.61 \%\right.$ and $\left.{ }^{11} \mathrm{~B} 80.39 \%\right)$ [4].

detectors are located around the target at angles 40,60, 90 and $120^{\circ}$, detector active area is $10 \times 10 \mathrm{~mm}$ and its depletion layer thickness is $0.3 \mathrm{~mm}, 1.512 \mathrm{MeV} \alpha$ beam hit the target uniformly in a circle of $0.5 \mathrm{~mm}$ radius. All simulation results are normalized to $10^{16}$ primary events.

In order to design the geometrical set-up of the detector system, we simulated the energy of out-going charge particles vs emitted angle $\theta$ (see Fig. 5) and then spectrum of the charged particle detected by the silicon detectors at different angle were also calculated. Fig. 6 show a simulated spectrum of the charge particle with silicon detector at $120^{\circ}$ after being convoluted with a energy resolution of $1.5 \%$. Results show that there are 4 protons produced by the ${ }^{10} B(\alpha, p){ }^{13} C$ corresponding to ground state and three excited state in residual nucleus. Furthermore, two contaminant reactions ${ }^{10} B\left(\alpha, d_{0}\right){ }^{12} C$ and ${ }^{11} B\left(\alpha, p_{0}\right){ }^{14} C$ are also simulated. In addition, for $\theta \geq 120^{\circ}$, energy of deuteron from contaminant reaction starts to be equal to that of the proton $p_{1}$ (see Fig. 5 and Fig. 6 ). These results are in good agreement with the experimental observation by Wilson at el [3]. The results also shows that further detector from the target the better energy resolution.

As mentioned, in the future experiment, our main interest is to re-measure the excitation function of the ${ }^{10} B(\alpha, p){ }^{13} C$ reaction. Due to the slowing down of the alpha by interaction with the target matterial, the energy of $\alpha$ particle induced the reaction is not always the same as initial one then determination the energy spectrum of such $\alpha$ for different target thickness is very important. By tracking the intial particle and the related process, we can simulate number of the reaction of interest and the relevant energy of the projectile at position of the reaction. Simulated energy spectrum of the $\alpha$ induced reaction for different target thickness 


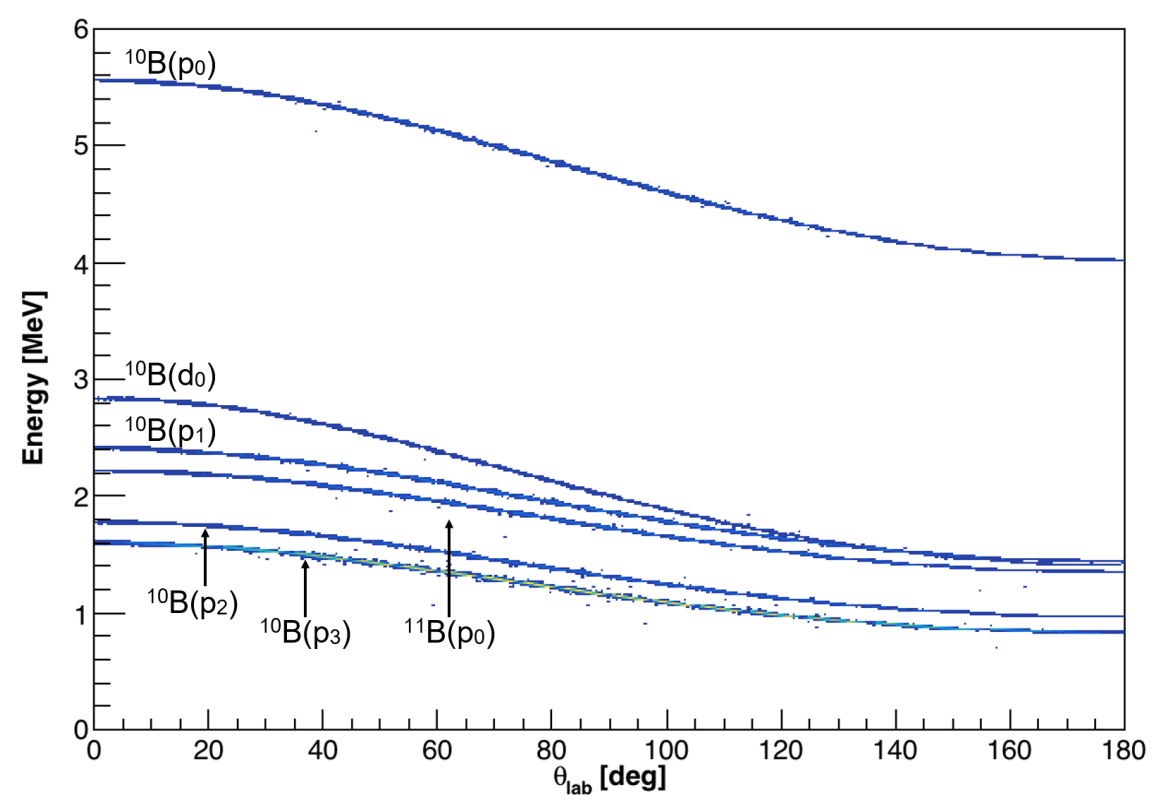

Figure 5: Out-going particle energy versa emitted angle $\theta$. Here, ${ }^{10} B\left(p_{0}\right),{ }^{10} B\left(p_{1}\right),{ }^{10} B\left(p_{2}\right),{ }^{10} B\left(p_{3}\right)$ denote proton produced by the reaction ${ }^{10} B(\alpha, p){ }^{13} C$ corresponding to ground state and excited states of the residual nucleus

are shown in Fig. 7. It is obvious that the energy of $\alpha$ inducing the reaction spread over a range of energy which depend on the thickness of the target, therefore, the reaction yield measured by experiment is intergation value. It means that for a thick target, we need to make a deconvolution to obtain the excitation function. This result play a very important role for analyzing experiment data.

\section{Conclusion}

Beside of the application study, with the small accelerator Pelletron, the nuclear-astrophyics study would be started in Vietnam. A Geant4-based code sucessfully simulated the ${ }^{10} B(\alpha, p){ }^{13} C$ at low energy which will help to design set-up of future experiment. This work is granted by Ministry of Sicence and Technology through the Physics Development Program Grant No. ĐTĐLCN.25/18. Authors would like to express sincere thanks for this valuable support.

\section{References}

[1] Shinya Wanjo, Hans-Thomas Janka and Shigeru Kubono, The Astrophysical Journal 729:41, 18 (2011)

[2] T. W. Bonner, Alfred A. Kraus, JR, J. B. Marion and J. P. Schiffer, Phys. Revl 102:5, 1348 (1956)

[3] Stephen J. Wilson, Phys. Rev 11:4, 1071 (1975) 

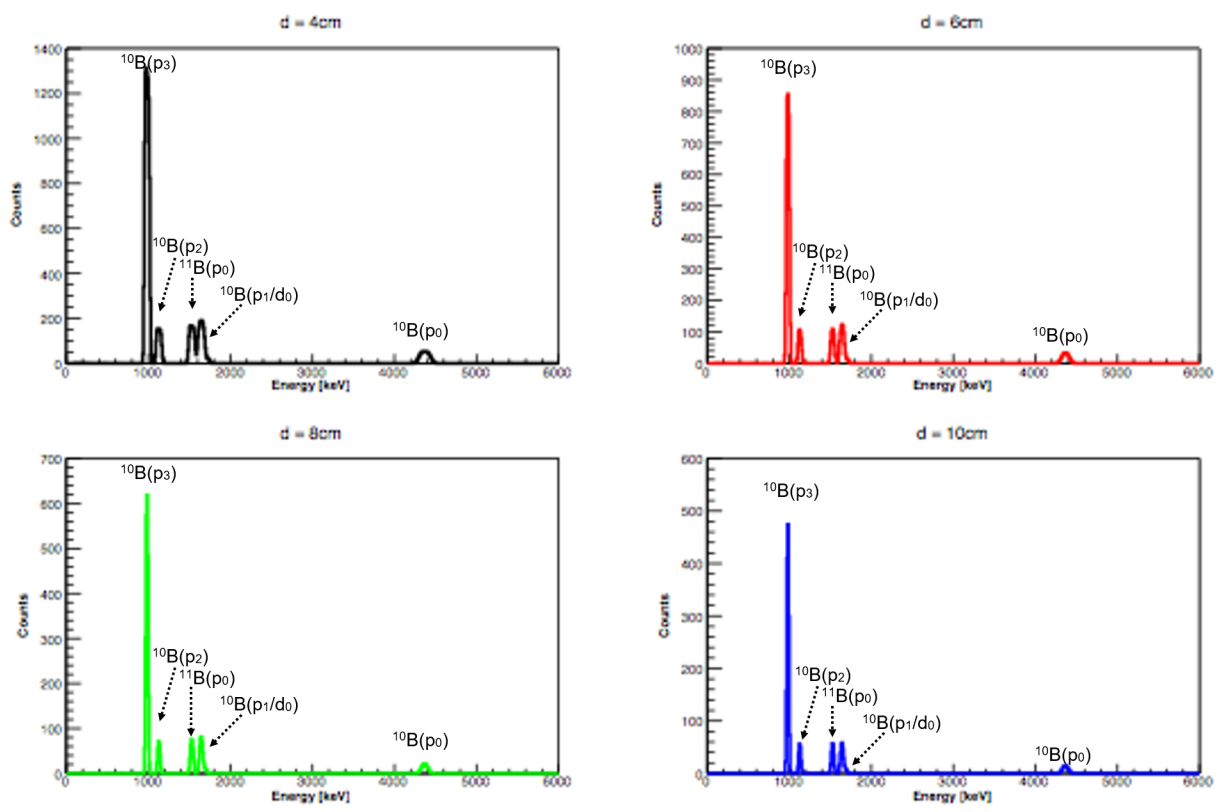

Figure 6: Spectrum of charged particle detected by silicon detector at $120^{\circ}$. Target thickness is $10 \mu \mathrm{m} / \mathrm{cm}^{2}$

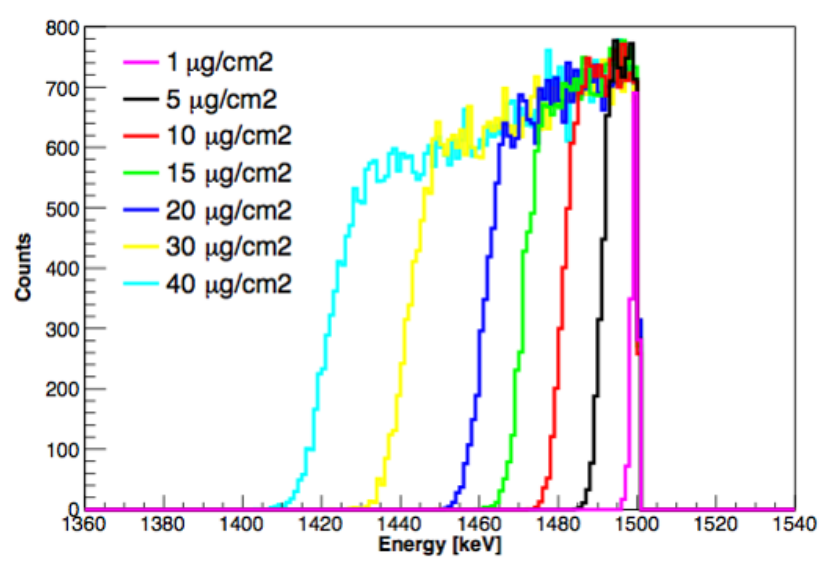

Figure 7: Energy spectrum of $\alpha$ which induce the reaction inside target with different target thickness

[4] H. Chen, X. M. Wang, L. Shao, J. R. Lieu, A. C. Yen, Wei-kan Chu, Nucl. Inst. and Meth. in Phys. Res. B 211, 1-6 (2003)

[5] A. Konning, S. Hilaire, S. Goriely, Talys 1.9 - User manual (2017) 